\title{
DESIGN FOR MASS ADAPTATION OF THE NEUROINTERVENTIONAL TRAINING MODEL HANNES WITH PATIENT-SPECIFIC ANEURYSM MODELS
}

\author{
Spallek, Johanna (1); Kuhl, Juliane (1); Wortmann, Nadine (1); Buhk, Jan-Hendrik (2); Frölich, \\ Andreas Maximilian (2); Nawka, Marie Teresa (2); Kyselyova, Anna (2); Fiehler, Jens (2); \\ Krause, Dieter (1)
}

1: Hamburg University of Technology; 2: University Medical Center Hamburg-Eppendorf

\begin{abstract}
A neurointerventional training model called HANNES (Hamburg ANatomical NEurointerventional Simulator) has been developed to replace animal models in catheter-based aneurysm treatment training. A methodical approach to design for mass adaptation is applied so that patient-specific aneurysm models can be designed recurrently based on real patient data to be integrated into the training system.

HANNES' modular product structure designed for mass adaptation consists of predefined and individualized modules that can be combined for various training scenarios. Additively manufactured, individualized aneurysm models enable high reproducibility of real patient anatomies. Due to the implementation of a standardized individualization process, order-related adaptation can be realized for each new patient anatomy with modest effort. The paper proves how the application of design for mass adaptation leads to a well-designed modular product structure of the neurointerventional training model HANNES, which supports quality treatment and provides an animal-free and patient-specific training environment.
\end{abstract}

Keywords: Product architecture, Additive Manufacturing, Patient-Specific Aneurysm Models, Training, Biomedical design

Contact:

Kuhl, Juliane

Hamburg University of Technology

Institute of Product Development and Mechanical Engineering Design

Germany

juliane.kuhl@tuhh.de

Cite this article: Spallek, J., Kuhl, J., Wortmann, N., Buhk, J.-H., Frölich, A.M., Nawka, M.T., Kyselyova, A., Fiehler, J., Krause, D. (2019) 'Design for Mass Adaptation of the Neurointerventional Training Model HANNES with PatientSpecific Aneurysm Models', in Proceedings of the 22nd International Conference on Engineering Design (ICED19), Delft, The Netherlands, 5-8 August 2019. DOI:10.1017/dsi.2019.94 


\section{INTRODUCTION}

An aneurysm is a pathological sacculation of a blood vessel that occurs in the brain area of $2-3 \%$ of the population (Fiehler, 2012). An intracranial aneurysm becomes life-threatening when a rupture in the aneurysm-weakened vessel wall occurs that is linked to a subarachnoid hemorrhage (Hacke and Poeck, 2010). If an aneurysm is detected, the primary aim of a treatment is to minimize the blood flow within the aneurysm. In order to practice the aneurysm treatment procedure and increase the likelihood of successful treatment, training for neuroradiologists is necessary (Neequaye et al., 2017). To simulate therapies of intracranial aneurysms, live rabbits, dogs, rodents, and pigs are used (Bouzeghrane et al., 2010).

Taking the 3Rs (replace, reduce, refine) defined by Russell and Burch (1959) for animal experimentation into account, animal-free aneurysm treatment training is desirable. Existing physical training models are not from adequate performance to replace the use of animals for endovascular treatment trainings. Patient-specific characteristics are insufficiently or not user-friendly and costeffectively replicated in current animal-free training models. Therefore, a new animal-free neurointerventional training model has been developed and verified in the project ELBE-NTM Development and Evaluation of a Patient-Based Neurointerventional Training Model.

The main focus in designing the neurointerventional training model is the patient-specific design of aneurysm models based on real patient anatomies. Aneurysm anatomies can be replicated true to the original. The aim of this paper is to examine how the application of a methodological approach to design for mass adaptation leads to a well-designed modular product structure of a neurointerventional training model that supports an animal-free and patient-specific training environment. The design process according to the design for mass adaptation and the resulting features of the innovative neurointerventional training model HANNES (Hamburg ANatomical NEurointerventional Simulator) are described. The primary focus of the training model is the integration of patient-specific aneurysms that replicate the real anatomy of a patient. Due to the implementation and recurrent application of a standardized individualization process, order-related adaptation can be realized for each new patient anatomy with only modest effort and reduced complexity. HANNES allows the simulation and the training of catheter-based interventions of intracranial aneurysms with real treatment instruments.

\section{BACKGROUND AND RESEARCH NEED}

Endovascular approaches for aneurysm treatment and current animal-free training models are presented in this section justifying the need for a neurointerventional training model.

\subsection{Endovascular treatment methods}

During an endovascular intervention of aneurysms, a catheter is advanced through the arteries under xray observation, starting at the femoral artery, until the intracranial aneurysm is reached. This minimal-invasive treatment approach is simulated with HANNES. Coil embolization, the remodeling technique with balloon or stent, and flow diverter implantation are possible treatment procedures. Selection depends on the shape, size and position of the aneurysm. The coil embolization is used for the majority of aneurysms with a closed aneurysm neck (Berlis, 2017). The aneurysm is filled with soft platinum spirals that enable coagulation of the blood in the aneurysm, so that the danger of rupturing is reduced (Hacke and Poeck, 2010). To prevent the coils from dropping into the carrier vessel, balloons or stents can be used for remodeling. Another relatively new approach for endovascular treatment is the use of a flow diverter which allows blood flow diversion and covers the entrance of the aneurysm (Simgen et al., 2012). Further instruments and techniques are constantly being developed, e.g. Woven EndoBridge (Berlis, 2017).

The endovascular approach is currently performed in many specialized centers in Germany, both in patients with acute subarachnoid hemorrhage and in patients who have been diagnosed with an aneurysm during other investigations. Patient-specific anatomy characteristics combined with the wide variety of treatment methods available necessitate training for many interventions. 


\subsection{Current training models for endovascular aneurysm treatment}

Knowing that the majority of interventional neuroradiologists have experienced animal-based training (Nawka et al., 2018), the motivation to replace animal experiments increases. Physical models exist with which the endovascular treatment of cerebral diseases, like aneurysm coiling, can be simulated. There are multiple small models that focus on a single vessel. They make it possible to place coils in an aneurysm and test the treatment, but they do not ensure the training in the complete treatment procedure.

A more detailed, holistic model is offered by the company Vascular Simulations, Inc. (New York, USA) that allows neuroradiologists to practice endovascular procedures. The model represents the vascular tree of the upper body and head, and includes a heart model made out of silicone and valves that simulate the pulsatile blood pressure. The company makes it possible to manufacture large vessels out of silicone specific to one patient's anatomy on the basis of imaging procedures (Vascular Simulations, 2018). However, to change the aneurysm model a gel inside the head model needs to be removed and re-filled, making the procedure of changing patient-specific aneurysm models complicated and time consuming.

The model EVE (EndoVascular Evaluator) of the company FAIN-Biomedical Inc. (Nagoya, Japan) enables endovascular intervention simulation. Customized human vessel models are generated by a patented modelling technology. It is possible to exchange all modules via special plug connectors to simulate different vascular diseases. EVE consists of a transparent vessel tree made out of silicone (FAIN-Biomedical 2018). Unfortunately, many silicon models have a high friction, so that the haptic when inserting a catheter is usually not comparable to a human body. The vessel replications are connected by adapters, but it is not clear whether inner edges are avoided for realistic replications.

Russ et al. (2015) designed a training model with additively manufactured cerebral models with different aneurysms. The post-printing cleaning process of material support, especially from small vessels, tortuous regions and aneurysms, is mentioned as a significant challenge by Ionita et al. (2014), indicating that high effort is required for patient-specific fabrication. Difficulties can occur, especially when dealing with a tortuous model or small vessels less than $2 \mathrm{~mm}$ (Ionita et al., 2014).

\subsection{Research need and requirements of a neurointerventional training model}

Current training models for endovascular aneurysm treatment presented in Section 2.2 make training without animals possible. Their similarity to real treatment can be evaluated for various aspects, such as the realistic replication of the whole vessel tree without inner edges. In particular, simple and costeffective adaptability of the model to the patient-specific training challenges is not part of most models. To improve the realism of treatment training and replace animal models in the long-term, a new neurointerventional training model is needed to fill the gaps identified. Users of the training model are mainly neuroradiologists, referred to here as physicians. Training for medical technical assistants can also be carried out with the training model.

To develop a new neurointerventional training model, the requirements of aneurysm treatment training were collected in interviews with physicians. The neurointerventional training model should replicate the whole relevant vessel tree, starting at the femoral artery, over the aorta and the carotid, until the cerebral vessels with the aneurysm is reached. A fluid simulating the blood should be at body temperature, with a human-like pulse. Multiple training scenarios in various aneurysm anatomies, vessel trees, treatment methods and procedures should be enabled. For the majority of training, the model should be used into an angiographic system for simulating the real treatment procedure with observation of the catheter in the vessel tree under x-ray. In some cases, an x-ray free training scenario is required to reduce radiation exposure and remove dependency on an angiographic system. Various vessel trees are required, especially ones with variation in the cerebral anatomy with aneurysm and carotid curves. Thus, the challenges for catheterizing the aneurysm in training can be varied and different patients can be simulated. The cerebral aneurysm model should be realized patient-specific based on real patient data, as individual anatomy challenges the neuroradiologists who have to choose between various medical instruments and their sizes. A large number of patient-original aneurysms will be modeled to increase the portfolio of aneurysm models. Thus, various patients' data varying especially in shape, orientation, diameter of the vessel, as well as aneurysm localization and size can be replicated. In addition, aneurysm model changes should be user-friendly and rapid during training. 


\section{DESIGN METHOD FOR THE NEUROINTERVENTIONAL TRAINING MODEL}

After clarifying requirements, the design phase of the training model is conducted, following a methodological approach for design for mass adaptation (Figure 1). This is a new approach by Spallek, based on |Spallek et al. (2016b, 2016c) and belongs to the Integrated PKT-Approach for Developing Modular Product Families (see (Krause and Gebhardt, 2018) for further information about the Integrated PKT-Approach). Mass adaptation stands for order-related product design done on a massive scale, meaning that individualization of an existing product is preplanned as much as possible and finally executed according to specific orders. Based on predicted individual needs of potential orders, a product is developed during the foundational phase that has the potential to be adapted to specific orders on a massive scale (Figure 1). A standardized individualization process targets to perform mass adaptation with modest effort. During order-related adaptation, some engineering activities are performed, resulting in an adapt-to-order environment, as defined by (Rudberg and Wikner, 2004). Due to a modular product structure, combined with preplanning and implementation of the standardized individualization process, order-related variants can be achieved with a manageable internal product and process complexity. The demand for patient-specific aneurysm models can be satisfied with the chosen design method for mass adaptation.

The modular product structure designed during the foundational phase of design for mass adaptation (Section 4) consists of predefined and individualized modules that can be combined for the various training scenarios. Predefined modules are modules designed for all predicted orders without any connection to a specific order. They can be standard, variant, or optional modules. In contrast, individualized modules are not completely defined; they need the special order requirements for individual patient anatomy for each order-related final design. A preparatory design of the individualized modules is made during the foundational phase, as it is fundament to the following order-related adaptation. The individualized modules, in this case the aneurysm model, are defined concerning their interfaces and individualization options. For the aneurysm models, it is possible to define a standardized process for order-related adaptation of each new patient-specific model, since the properties of the vessel anatomies are highly predictable, as human anatomies closely resemble each other.

The order-related adaptation (Section 5) follows the prepared standardized individualization process for each new patient's anatomy data. Thus, recurrent adaptations are accepted and executed according to specific orders with modest effort.

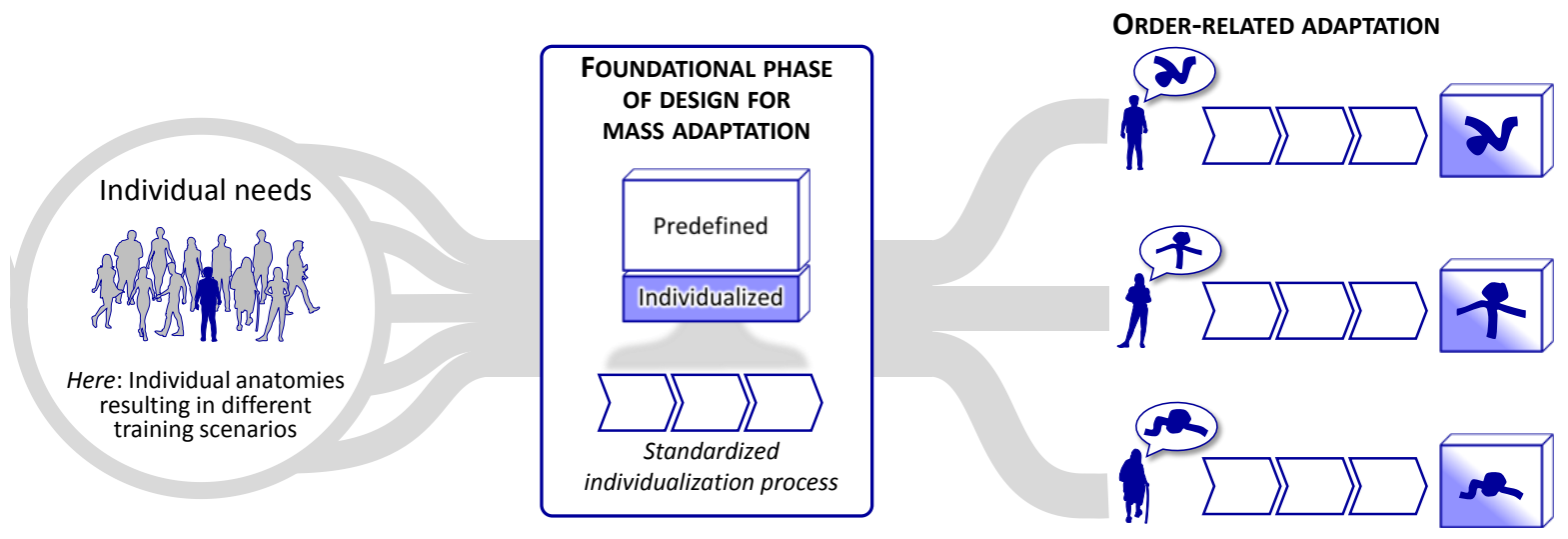

Figure 1: Design for mass adaptation with a standardized individualization process

\section{FOUNDATIONAL PHASE OF DESIGN FOR MASS ADAPTATION}

The foundational phase of design for mass adaptation starts by analyzing the predicted need, benefit and potential of mass adaptation. After designing a modular product structure for mass adaptation and defining predefined and individualized modules, the preparatory design for the individualized modules is detailed. Concepts for the individualized modules and possible solutions for the standardized individualization process are developed and evaluated. The preparatory design of the individualized modules with their interfaces is made for the preferred concept. The standardized individualization process is detailed and elaborated. The steps of HANNES' foundational phase of design for mass adaptation are presented in the following section. 


\subsection{Analysis step}

The first step in the foundational phase is to analyze the individualization need and the expected individual demands. The required product properties are analyzed for their relevance to prospective individual adaptable design. With the aid of physicians, it was determined that individualization is necessary (otherwise predefined product variants are preferred for complexity management) while mass adaptation is possible in a preparatory way (otherwise single-unit production are better implemented). Individualization options are established as the availability of individualized aneurysm models designed according to patient medical data.

\subsection{Design of the modular structure of HANNES}

The module definition in design for mass adaptation follows the main idea and tools of the design for variety of Kipp (2012), as part of the Integrated PKT-Approach for a product family with predefined product variants, and is combined with explicit considerations for mass adaptation. The main idea here is to achieve one-to-one binding of order-related product properties to individualized components and the decoupling of individualized to predefined components. The developed modular structure of HANNES consists of standard, variant and individualized modules. They are marked in Figure 2 with different colors, using the main idea of a Module Interface Graph (MIG) as introduced in (Blees et al., 2010). Standard components (white) are used in every product variant of the product family. For the demonstrator, the aorta, base frame, fluid system and control unit are used in every training scenario. To achieve different training scenarios, variant (grey) and individualized components (blue) are added to the training model. Variant components mean that they are predefined and designed independently of a specific order, whereas individualized components are specified concerning order-related requirements. At this point, the term patient-based is introduced, which means that variant or standard modules are designed according to an existing anatomy of a patient.

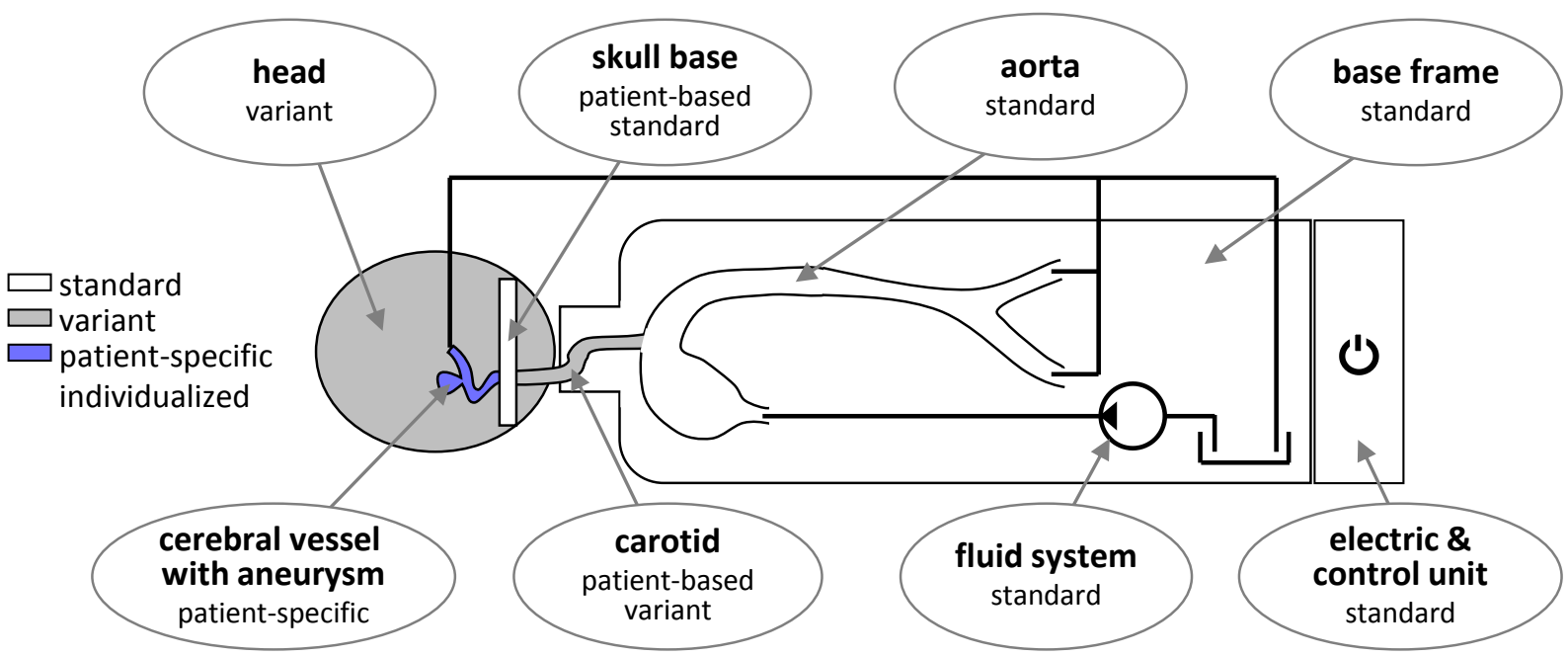

Figure 2: Modular product structure of the neurointerventional training model HANNES

\subsection{Preparatory design of individualized modules and individualization processes}

An essential step in the foundational phase of design for mass adaptation is preparatory design of the individualized modules and related individualization processes. The individualized modules are specified concerning their offered individualization options and their interfaces to other modules. The individualization process for the final detail design, fabrication, and assembly of an individualized module is prepared as much as possible so that it can be undertaken recurrently with minor effort. The vessel anatomies have highly predictable properties in their anatomies, so that an individualization process for the aneurysm models is developed and standardized.

The preparatory design is an iterative process for several concepts that are compared to each other to finally select the preferred one. The high coupling between individualized design and individualization process, in particular for interface definition and automatization degree, is the reason, why both product and process should be considered when reducing internal complexity. The final concept for aneurysm models and its design and process is described in more detail in the following section. 


\subsubsection{Cerebral vessel model with aneurysm}

The inner shapes of the hollow aneurysm models simulate different patient-original cerebral vessel anatomies with intracranial aneurysms acquired in angiographic measurements. There are no inner edges at the transition of the skull base to the cerebral aneurysm model. When specifying the aneurysm model, the adapter is added in a way that the aneurysm is placed in the true to original position inside the head only by correctly connecting the individualized aneurysm model to the standard skull base model (Figure 3).

The aneurysm model can be prepared for mass adaptation. Individualization options exist for anatomy, meaning orientation, shape and diameter of the vessel, choice of reproduced arteries, aneurysm localization and size, length of reproduced artery, and number of flow outlets. Further options include wall thickness for strength and stability and the material properties. Boundaries of the individualization are given by the head model size and the available material choice.

Additive manufacturing (AM) technologies are used to fabricate aneurysm models due to the advantages in huge geometrical freedom combined with high production flexibility, especially for low lot sizes. A large range of plastic materials and technologies are available, which differ in material properties, e.g. elasticity, transparency, and the feasibility of support removal in the inner hollow vascular structure. A comprehensive comparison of AM materials for plastic vessel models is presented in Spallek (2016a). Models fabricated by Fused Deposition Modeling (FDM) offer replication at acceptably high accuracy (Frölich et al., 2015). Higher performance in training can be achieved using models made of flexible materials. Two flexible materials are preferred and have increased resolution compared to FDM (Figure 4left): One is made by Stereolithography using a material called "Flexible" (Formlabs machine). This choice has the advantage that no inner support structures are needed during the manufacturing, enabling a smooth and edge-free inner surface. The second is made with the Multi Jet Modelling technology using a material called "TangoPlus FLX930" (Connex machine). This material is more flexible but the removal of support structures is quite time consuming. The selection of material depends on the anatomy and training scenarios.

\subsubsection{Standardized individualization process of the aneurysm model}

The individualization process is prepared to perform each order-related adaptation with reduced process complexity. The process is characterized by data acquisition, design of specification, fabrication, and assembly. Each step is analyzed for whether a specific order is necessary for performing the step. If the step is unrelated to specific orders it should be predefined separately. All order-related steps are prepared predictively. They are subsequently standardized and summarized in a standardized individualization process which is passed for each order (|Spallek et al., 2016b, 2016c).

The standardized individualization process of the mass adaptation of the aneurysm model (Figure 3) begins with the acquisition of medical data by a three-dimensional angiographic scan of the aneurysm. The data is segmented and relevant vessels are retained. Model specifications, like the positioning of the adapters or the definition of the wall thickness, take place. After finishing this step, the aneurysm model is manufactured by AM in flexible plastic materials. After completion of post-processing, e.g. the removal of the support structures, the aneurysm model is available for training applications.

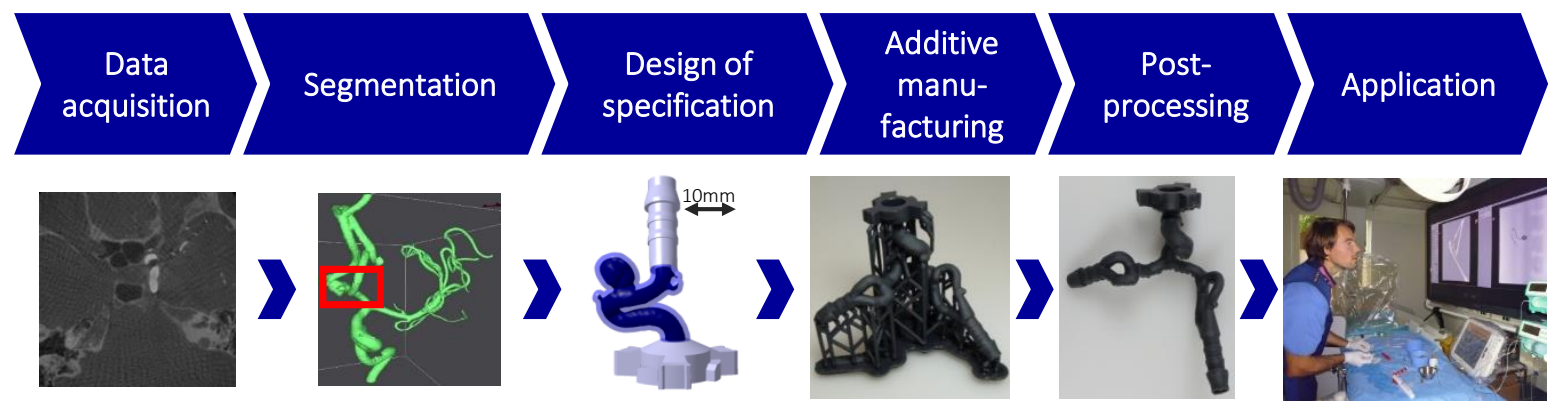

Figure 3: Standardized individualization process for the mass adaptation of patient-specific aneurysm models, adapted to Spallek et al. (2016b)

The design of specification, as one step of the standardized individualization process (Figure 3), can be implemented in different ways, e.g. through classic CAD software, special tool kits for vascular modeling, or tools for AM. The aim of the preparatory design of the individualization process is to prepare as many standardizations and automations as possible for complexity management while 
enabling multiple individualization options. Therefore, it is important to define who will perform each step. The choice about the tool used for design for specification depends heavily on the person performing it and their skills and abilities. As mentioned, the design of the individualized module is strongly coupled to the standardized individualization process, as it recurs for each new aneurysm model. This means that when choosing another design, e.g. to offer more individualization options, another process and another implementation, e.g. the chosen tool for the design of specification, can be necessary.

\subsection{Detail design of predefined modules}

Besides preplanning the individualized modules, the foundational phase of design for mass adaptation concludes the detail design of all predefined modules and their fabrication and supply chain.

\subsubsection{Variant components for creating different training scenarios}

The patient-specific aneurysm model is placed inside the head model, for which two variants currently exist. One variant of the head model, made out of ABS polymer material, is intended to be used in an $\mathrm{x}$-ray environment (Figure 4). The second head variant is designed for an $\mathrm{x}$-ray free training scenario. Instead of an imaging with x-rays, an optical system with a camera is used for observation of the catheter inside the transparent aneurysm model. Software programmed for the purpose simulates an x-ray environment (Schmidt 2018).

Variant carotid models are used for completing the patient-based vessel tree. They vary in the intensity of their curves (Figure 4, right), so that patients of different ages and varying difficulty in catheterizing the cerebral vessels can be simulated. The shape of the carotid is designed to be patient-based but in a defined number of variants. They are designed and manufactured similar to the aneurysm models, so that patient-specific carotid models can be made in the future.

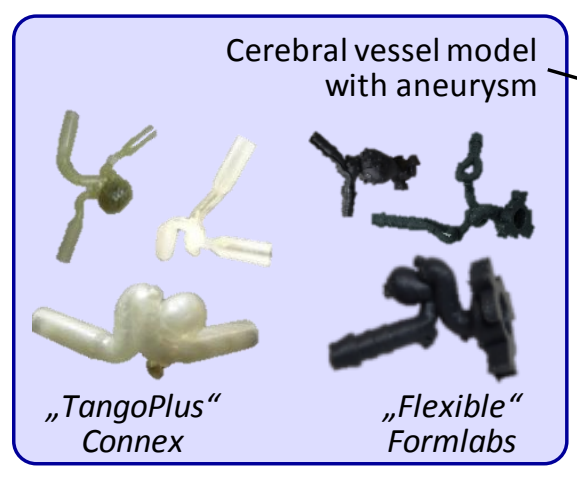

Figure 4: Patient-specific, flexible aneurysm model and variant carotid models integrated into the demonstrator, with the head for $x$-ray environment

\subsubsection{Standard modules shared among all training scenarios}

A standard skull base and an aorta model are implemented to complete the relevant vessel tree for the aneurysm treatment training, which are identical for every training scenario. The aorta is made by United Biologics, Inc. (Santa Ana, California, USA) out of silicon. The silicon does not replicate the behavior of the human vessel system very accurate, because it is much stickier and does not allow the physician to insert the catheter exactly in the same manner as in a real treatment. The inner structure of the skull base is a patient-based standard design related to one patient's original medical data. The exact outlook of the passage varies from patient to patient but the general anatomical course is the same for everyone, so there is no urgent need to design individualized skull base models.

A blood circulation system is added for even better realism. The fluid system, which is the same for every training scenario, consists of a pump and a valve to replicate the heartbeat as well as a tank for the fluid. To simulate the blood, water with some soap or glycerol is used and can be adjusted to the human body temperature of $37^{\circ} \mathrm{C}$. With different valves and throttles the pressure inside the system can be adjusted. The flow rate can be adapted to the needs of the training scenario. Other parameters 
are controlled by a control unit, e.g. the pulse of the system. The base frame enables the assembly of the individualized, variant and standard modules in a compact demonstrator.

Further adaptations of the standard modules are planned to steadily improve the demonstrator. Due to the current, unrealistic haptic of the bought aorta, the design and fabrication of an aorta with an additional interface between the distal and proximal aorta parts are planned, so that a variation of the aorta to demonstrate different age structures of patients can be implemented. In addition, how to improve the haptic behavior of the vessel models in general is being analyzed to minimize the difference between the model and reality even further.

\section{ORDER-RELATED ADAPTATION}

The order-related adaptation occurs recurrently for each new aneurysm anatomy and is achieved according to the deliverables of the foundational phase of design for mass adaptation (compare Figure 1). With the prepared standardized individualization process of the aneurysm model (Section 4.3.2), it is possible to replicate any cerebral aneurysm for which suitable medical data is available (Figure 5). More than 30 different aneurysm models exist, which include aneurysms in different sizes, positions and executions. Further models are planned so that the portfolio of patient-specific aneurysm models is continuously extended due to the standardized individualization process.

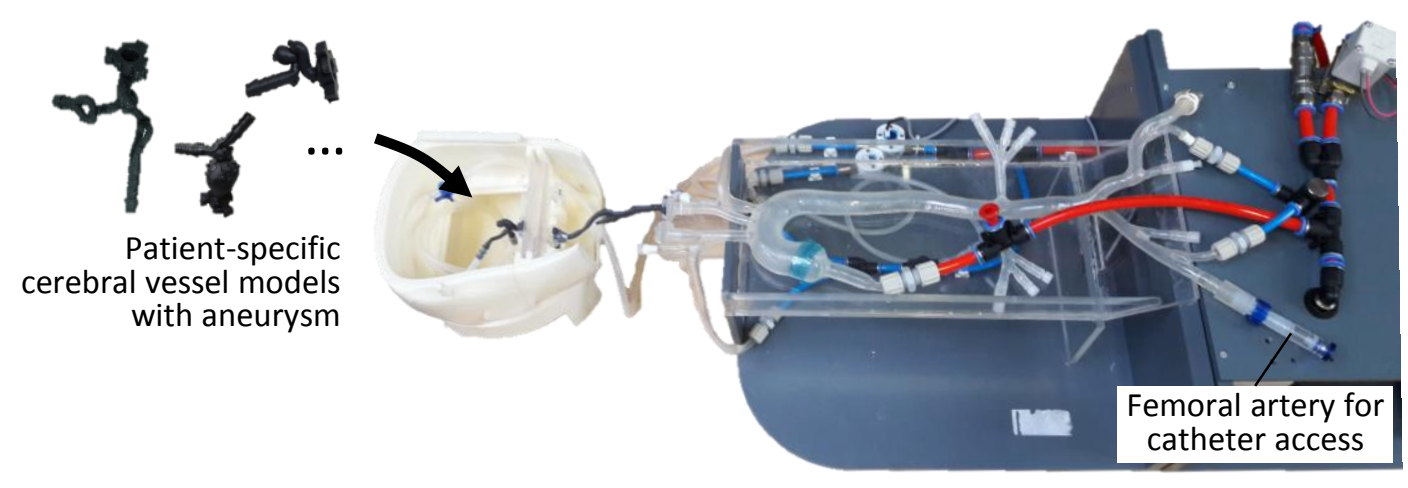

Figure 5: HANNES demonstrator contains the potential to be adapted to patient-specific aneurysm data on a massive scale

The demonstrator HANNES is already used in training for aneurysm treatment with patient-specific aneurysm models. Figure 6 shows HANNES during training procedures in the clinical environment with x-ray application. All aneurysm models can be rapidly assembled in a user-friendly manner within the demonstrator including that no inner edges occur at the interface of one component to another. In angiographic imaging there seems to be one coherent structure, which creates a highly realistic training environment (Figure 6 (b)). In addition, the adapter enables their correct positioning and orientation.
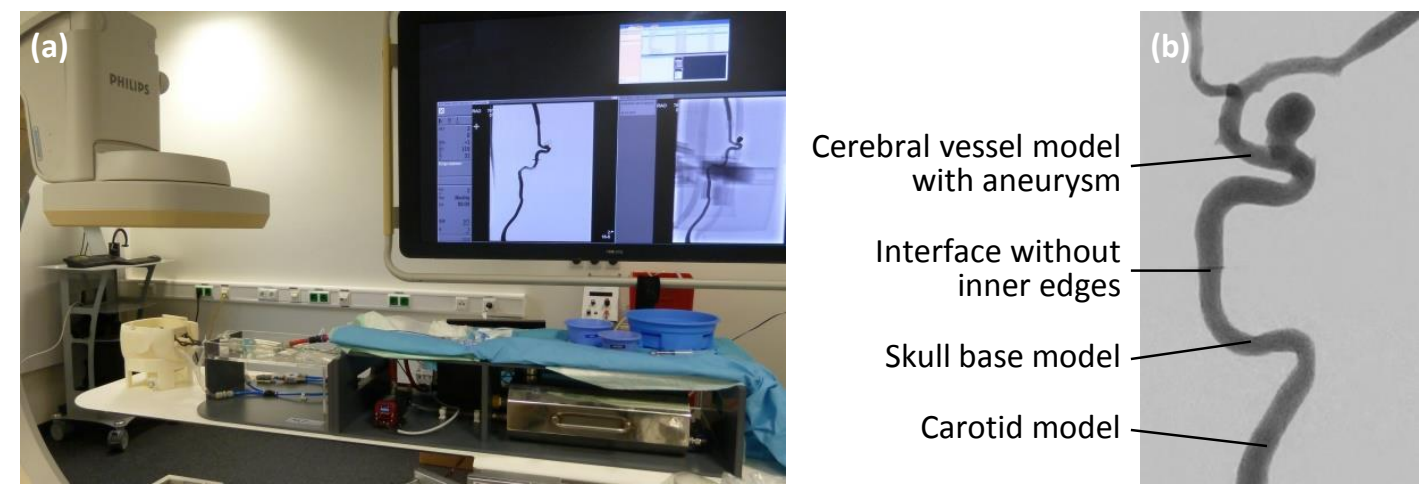

Figure 6: Clinical use of the HANNES demonstrator (a) and the resulting digital subtraction angiography of a vessel tree with aneurysm model (b)

Since the dimensions of the vessel tree regarding the diameters and length mimic the human body, training in realistic anatomies is possible and the physicians can use original treatment instruments 
with catheter access at HANNES' femoral artery (Figure 5). This means that not only the prevention of animal models is possible but also training closer to reality since dimensions fit to the human body.

\section{SUMMARY AND OUTLOOK}

The demonstrator HANNES has been developed to replace animal models in cerebral aneurysm treatment training. Thus, training for the treatment of intracranial aneurysms can be carried out with real treatment instruments, using a vessel tree model that simulates human vessels.

Patient-specific design of the aneurysm model is important for aneurysm treatment training, thus the mass adaptation of the aneurysm model is enabled by HANNES so that further aneurysm models will be individualized for new patient cases. A methodological approach for design for mass adaptation is applied to reduce the order-related complexity for each individual order and to enable individual product design on a massive scale. During the foundational phase of design for mass adaptation, the product and process structure of HANNES are designed. Predefined modules are completely designed in detail while the individualized modules are developed as preparatory design. A standardized individualization process is established for the aneurysm models to facilitate the flexibility required for adaptation and fabricating new patient-specific aneurysm models with the aid of AM. Accordingly, the aneurysm models can be adapted in the order-related adaptation phases based on real patient data.

HANNES' modular product architecture enables flexible use by configuring different training scenarios and making internal complexity controllable. Training scenarios using HANNES can be adapted to real cases. The main idea is to prevent animal models in aneurysm treatment training. Experienced and new physicians can train the catheter-based treatment and make themselves familiar with the procedure. The modular structure not only allows the configuration of training scenarios but also makes the HANNES demonstrator resilient to changes since the structure allows the adaptation of modules without affecting others. In addition, the platform can be expanded and other vessel diseases, e.g. strokes, can be added within a short development time. Furthermore, the planning or follow-up of real aneurysm treatments can be discussed with the help of HANNES.

This paper demonstrates the application of a design method for mass adaptation with the implementation of a standardized individualization process. The preparation of a standardized individualization process supports complexity management so that an infinitely high external product variety can be achieved with manageable internal complexity. An innovative demonstrator of a neurointerventional training model that supports medical progress and treatment quality while offering animal-free and patient-specific training setups can be achieved and expanded in the future.

\section{ACKNOWLEDGEMENTS}

The authors would like to thank the Federal Ministry of Education and Research - BMBF for funding the project ELBE-NTM, project number 031 L0068A. We would also like to thank the whole project team, especially our partners at the Institute of Microsystems Technology at Hamburg University of Technology and the neurointerventional training participants for their feedback.

\section{REFERENCES}

Berlis, A. (2017), “Endovaskuläre Behandlung von Hirn-Aneurysmen Hirn- Aneurysma/ Angiom”, in: Berlis, A. and Schneebauer, C. (Eds.), Kompendium für Betroffene, Angehörige und Interessierte, Association for brain aneurysm sufferers, Der Lebenszweig e.V. (own publishing house), http://www.hirnaneurysma.de/files/redakteur/Kompendium\%20Testseiten.pdf, accessed date 29.11.2018

Blees, C., Jonas, H. and Krause, D. (2010), "Development of modular product families", 12th International DSM Conference, Cambridge, 22-23 July 2010, Carl-Hanser-Verlag, Munich, pp.169-182

Bouzeghrane, F., Naggara, O., Kallmes, D.F., Berenstein and A. and Raymond, J. (2010), "In vivo experimental intracranial aneurysm models: a systematic review”, American Journal of Neuroradiology, March 2010, Vol. 31 No. 3, pp. 418-423, https://doi.org/10.3174/ajnr.A1853

FAIN-Biomedical (2018), Endovascular Surgery Training-Evaluation-Simulation EVE EndoVascular Evaluator, http://fain-biomedical.com/fbm_wp/wp-content/themes/fbm_ns/images/pdf/eve_fbm_e.pdf, accessed date 29.11.2018

Fiehler, J. (2012), "Nicht rupturierte intrakranielle aneurysmen: wann suchen, wann behandeln? " RöFo : Fortschritte auf dem Gebiete der Röntgenstrahlen und der Nuklearmedizin, Vol. 184 No. 2 , Georg Thieme Verlag KG, Stuttgart, pp. 97-104, https://doi.org/10.1055/s-0031-1281984 
Frölich, A.M.J., Spallek, J., Brehmer, L., Buhk, J.-H., Krause, D., Fiehler, J. and Kemmling, A. (2015), “3D printing of intracranial aneurysms using fused deposition modeling offers highly accurate replications", AJNR American Journal of Neuroradiology, originally published online on August 20, 2015, https://doi.org/10.3174/ajnr.A4486

Hacke, W. and Poeck, K. (2010), Neurologie: Mit 83 Tabelle, 13th completely revised edition, SpringerMedizin-Verlag, Heidelberg, https://doi.org/10.1007/978-3-662-46892-0

Ionita, C.N., Mokin, M., Varble, N., Bednarek, D.R., Xiang, J., Snyder, K.V., Siddiqui, A.H., Levy, E.I., Meng, H. and Rudin, S. (2014), "Challenges and limitations of patient-specific vascular phantom fabrication using 3D Polyjet printing”, SPIE--the International Society for Optical Engineering, Vol. 9038 p. 90380M., https://doi.org/10.1117/12.2042266

Kipp, T. (2012), “Methodische unterstützung der variantengerechten produktgestaltung”.Dissertation TU Hamburg-Harburg, TuTech Verlag Hamburg, Hamburger Schriftenreihe Produktentwicklung und Konstruktionstechnik, Band 4, ISBN 978-3-941492-47-9

Krause, D. and Gebhardt, N. (2018), Methodische Entwicklung modularer Produktfamilien - Hohe Produktvielfalt beherrschbar entwickeln, Springer-Verlag, Hamburg, 2018, https://doi.org/10.1007/978-3662-53040-5

Krause, D., Spallek, J., Blees, C. and Kipp, T. (2018), “Modulare Produktstrukturierung”, In: Rieg, F. and Steinhilper, R. (Ed.), Handbuch Konstruktion, Carl Hanser Verlag, München, pp. 719-741, https://doi.org/10.3139/9783446434035

Nawka, M. T., Fiehler, J., Spallek, J., Buhk, J.-H. and Frölich, A.M. (2018), “Current status of training environments in neuro-interventional practice: are animal models still contemporary?", Journal of Neurointerventional Surgery, 2018 Jul 26, https://doi.org/10.1136/neurintsurg-2018-014036

Neequaye, S. K., Aggarwal, R.,Van Herzeele, I., Darzi, A. and Cheshire, N.J. (2017), "Endovascular skills training and assessment”, Journal of vascular surgery, Vol.46, pp. 1055-1064, https://doi.org/10.1016/j.jvs.2007.05.041

Rudberg, M. and Wikner, J. (2004), "Mass customization in terms of the customer order decoupling point", Production Planning \& Control Vol. 15 No. 4, S. 445-458. https://doi.org/10.1080/0953728042000238764.

Russ, M., O’Hara, R., Setlur Nagesh, S. V., Mokin, M., Jimenez, C., Siddiqui, A., Bednarek, D., Rudin, S. and Ionita, C. (2015), "Treatment planning for image-guided neuro-vascular interventions using patientspecific 3d printed phantoms”, Proceedings of SPIE--the International Society for Optical Engineering 9417

Russell, W.M.S. and Burch, R.L. (1959), The Principles of Humane Experimental Technique, Methuen, London Schmidt, P. (2018), "Konzeptentwicklung einer röntgenfreien trainingsumgebung für aneurysmabehandlungen", Master Thesis at the Institute of Product Development and Mechanical Engineering Design, Hamburg University of Technology

Simgen, A., Junk, D. and Reith, W. (2012), "Flow diverter. eine neue therapiemöglichkeit für intrakranielle aneurysmen", Der Radiologe, Vol. 52 No. 12, pp. 1118-24

Simbionix USA Corporation (2017), ANGIO Mentor. The Most Advanced Endovascular Training, URL: http://simbionix.com/simulators/angio-mentor/, accessed date 29.11.2018

Spallek, J., Frölich, A., Buhk, J.H., Fiehler, J. and Krause, D. (2016a), “Comparing technologies of additive manufacturing for the development of vascular models", Fraunhofer Direct Digital Manufacturing Conference DDMC, Fraunhofer Verlag, Berlin.

Spallek, J. and Krause, D. (2016b), "Process types of customisation and personalisation in design for additive manufacturing applied to vascular models", 26th CIRP Design Conference, Vol. 50, Procedia CIRP, Elsevier, pp. 281-286, http://doi.org/10.1016/j.procir.2016.05.022

Spallek, J., Sankowski, O. and Krause, D. (2016c), "Influences of additive manufacturing on design processes for customised products", 14th International Design Conference - DESIGN 2016, Cavtat, Dubrovnik, Croatia, pp. 513-522, https://doi.org/10.13140/RG.2.1.1112.4080

Vascular Simulations (2018), Optimize Your Performance, https://vascularsimulations.com/technology/, accessed date 29.11.2018 